\title{
LIFTINGS AND THE CONSTRUCTION OF STOCHASTIC PROCESSES
}

\author{
BY
}

DONALD L. COHN

\begin{abstract}
It is shown that if the continuum hypothesis holds, then the use of liftings to construct modifications of stochastic processes can replace measurable processes with nonmeasurable ones. The use of liftings to choose the paths, rather than the random variables, of a stochastic process is investigated.
\end{abstract}

1. Definitions and introduction. Let $K$ be a compact metric space, let $\mathscr{B}(K)$ be the $\sigma$-algebra of Borel subsets of $K$, let $(\Omega, \mathcal{Q}, P)$ be a probability space, and let $T$ be a nonempty set of real numbers. A stochastic process on $(\Omega, \mathcal{Q}, P)$ with values in $K$ and parameter space $T$ is an indexed family $\left\{X_{t}\right\}_{t \in T}$ of $K$-valued functions on $\Omega$ that are measurable with respect to $\mathbb{Q}$ and $\mathscr{B}(K)$. Recall that a stochastic process $\left\{X_{t}\right\}_{t \in T}$ is separable if there is a $P$-null subset $N$ of $\Omega$ and a countable subset $D$ (called a separating set) of $T$ such that for each $\omega$ in $\Omega-N$ the graph of the restriction of the path $t \mapsto X_{t}(\omega)$ to $D$ is dense in the graph of $t \mapsto X_{t}(\omega)$. Recall also that the process $\left\{X_{t}\right\}_{t \in T}$ is Borel measurable if the parameter space $T$ is a Borel subset of $\mathbf{R}$ and the map $(t, \omega) \mapsto X_{t}(\omega)$ is measurable with respect to the product $\sigma$-algebra $\mathscr{B}(T) \times \mathscr{Q}$ (here $\mathscr{B}(T)$ is the $\sigma$-algebra of Borel subsets of $T$ ). Likewise the process $\left\{X_{t}\right\}_{t \in T}$ is Lebesgue measurable if the parameter space $T$ is a Lebesgue measurable subset of $\mathbf{R}$ and the map $(t, \omega) \mapsto X_{t}(\omega)$ is measurable with respect to the completion of $\mathscr{T}_{\lambda}(T) \times \mathbb{Q}$ under the product measure $\lambda \times P$ (here $\Re_{\lambda}(T)$ is the $\sigma$-algebra of Lebesgue measurable subsets of $T$ and $\lambda$ is Lebesgue measure). Finally recall that processes $\left\{X_{t}\right\}_{t \in T}$ and $\left\{Y_{t}\right\}_{t \in T}$ on a common probability space $(\Omega, \mathcal{Q}, P)$ are modifications of one another if $P\left(X_{t} \neq Y_{t}\right)=0$ holds for each $t$ in $T$, and that processes $\left\{X_{t}\right\}_{t \in T}$ and $\left\{Y_{t}\right\}_{t \in T}$ on possibly different probability spaces are versions of one another if they have the same finite dimensional distributions.

For a finite or $\sigma$-finite measure space $(E, \mathcal{E}, \mu)$ let $\mathfrak{L}^{\infty}(E, \mathcal{E}, \mu)$ be the vector space of bounded real-valued $\mathcal{E}$-measurable functions on $E$, and let $L^{\infty}(E, \mathcal{E}, \mu)$ be the quotient space formed by identifying functions in $\mathfrak{L}^{\infty}(E, \mathcal{E}, \mu)$ that agree $\mu$-almost everywhere. For functions $f$ and $g$ on $E$ we

Received by the editors November 17, 1977.

AMS (MOS) subject classifications (1970). Primary 46G15, 60G05. 
shall write $f \equiv g$ to indicate that $f$ and $g$ agree $\mu$-almost everywhere, and $f=g$ to indicate that $f$ and $g$ agree everywhere. We shall occasionally denote by $f^{\sim}$ the class in $L^{\infty}(E, \mathcal{E}, \mu)$ determined by the function $f$. A lifting of $\mathfrak{L}^{\infty}(E, \mathcal{E}, \mu)$ is a map $\rho: \mathfrak{L}^{\infty}(E, \mathcal{E}, \mu) \rightarrow \mathfrak{L}^{\infty}(E, \mathcal{E}, \mu)$ that satisfies

(i) $\rho(a f+b g)=a \rho(f)+b \rho(g)$,

(ii) $\rho(f g)=\rho(f) \rho(g)$,

(iii) $\rho(1)=1$,

(iv) $\rho(f)=\rho(g)$ if $f \equiv g$, and

(v) $\rho(f) \equiv f$

for all $f, g$ in $\mathfrak{L}^{\infty}(E, \mathcal{E}, \mu)$ and all $a, b$ in $\mathbf{R}$. In view of condition (iv), a lifting induces a map from $L^{\infty}(E, \mathcal{E}, \mu)$ to $\mathfrak{L}^{\infty}(E, \mathcal{E}, \mu)$; it is easy to check that this correspondence provides a bijection between the set of liftings of $\mathcal{L}^{\infty}(E, \mathcal{E}, \mu)$ and the set of algebra homomorphisms of $L^{\infty}(E, \mathcal{E}, \mu)$ into $\mathcal{L}^{\infty}(E, \mathcal{E}, \mu)$ that preserve the identity and map each equivalence class in $L^{\infty}(E, \mathcal{E}, \mu)$ to one of its members. It is known that if the finite or $\sigma$-finite measure space $(E, \mathcal{E}, \mu)$ is complete, then there is a lifting of $\mathfrak{L}^{\infty}(E, \mathcal{E}, \mu)$ (see [6] and the references contained therein).

We shall be dealing with an extension of the notion of lifting to functions that are not real-valued. Consider the following special case of a construction given by $\mathrm{A}$. and $\mathrm{C}$. Ionescu Tulcea [5]. Let $(E, \mathcal{E}, \mu)$ be a measure space for which $\mathcal{L}^{\infty}(E, \mathcal{E}, \mu)$ has a lifting, say $\rho$. Let $\mathfrak{T}(E, \mathcal{E}, \mu, K)$ be the set of all $K$-valued functions on $E$ that are measurable with respect to $\mathcal{E}$ and $\mathscr{B}(K)$. Let $C(K)$ be the (real) Banach algebra consisting of all continuous realvalued functions on $K$. Given a point $t$ in $E$ and a function $F$ in $\Re(E, \mathcal{E}, \mu, K)$, consider the real-valued algebra homomorphism on $C(K)$ defined by $g \mapsto \rho(g \circ F)(t)$. According to elementary Banach algebra theory, this homomorphism is given by evaluation at some point in $K$; call this point $\rho^{\prime}(F)(t)$. This gives a function $\rho^{\prime}(F)$ from $E$ to $K$ that is easily seen to be $\mathcal{E}$-measurable. The map $\rho^{\prime}$ from $\Re(E, \mathcal{E}, \mu, K)$ to $\Re(E, \mathcal{E}, \mu, K)$ that takes $F$ to $\rho^{\prime}(F)$ then satisfies (for all $F, G$ in $\Re(E, \mathcal{E}, \mu, K)$ and all $g$ in $C(K))$

(1) $g \circ \rho^{\prime}(F)=\rho(g \circ F)$,

(2) $\rho^{\prime}(F) \equiv F$, and

(3) if $F \equiv G$, then $\rho^{\prime}(F)=\rho^{\prime}(G)$.

Furthermore $\rho^{\prime}$ is uniquely determined by these conditions. The map $\rho^{\prime}$ will be called the lifting of $\Re(E, \mathcal{E}, \mu, K)$ induced by $\rho$.

Now suppose that the probability space $(\Omega, \mathbb{Q}, P)$ is complete, that $\rho$ is a lifting of $\mathfrak{L}^{\infty}(\Omega, \mathcal{Q}, P)$, that $\rho^{\prime}$ is the lifting of $\Re(\Omega, \mathcal{Q}, P, K)$ induced by $\rho$, and that $T$ is a subinterval of $\mathbf{R}$. A. and C. Ionescu Tulcea [5] have shown that if $\left\{X_{t}\right\}_{t \in T}$ is a stochastic process on $(\Omega, \mathcal{Q}, P)$ with values in $K$, then $\left\{\rho^{\prime}\left(X_{t}\right)\right\}_{t \in T}$ is a separable modification of $\left\{X_{t}\right\}_{t \in T}$. We show that this construction need not produce a measurable process. More precisely, we shall 
show that if the continuum hypothesis holds, then there is a complete probability space $(\Omega, \mathcal{Q}, P)$, a lifting $\rho$ of $\mathfrak{L}^{\infty}(\Omega, \mathcal{Q}, P)$, and a $[-\infty, \infty]-$ valued stochastic process $\left\{X_{t}\right\}_{t \in T}$ on $(\Omega, \mathbb{Q}, P)$ that is Borel measurable but for which the modification $\left\{\rho^{\prime}\left(X_{t}\right)\right\}_{t \in T}$ is not even Lebesgue measurable. This proof depends on a construction due to R. M. Dudley [2] and a theorem due to G. Mokobodzki [9]; the details are given in $\$ 2$ below.

In $\$ 3$ we change our point of view, and instead of applying liftings of $\mathfrak{L}^{\infty}(\Omega, \mathcal{Q}, P)$ to the random variables $X_{t}$, producing random variables $\rho^{\prime}\left(X_{t}\right)$, we apply liftings of $\mathfrak{L}^{\infty}\left(T, \mathscr{T}_{\lambda}, \lambda\right)$ (where $\mathscr{T}_{\lambda}$ is the $\sigma$-algebra of Lebesgue measurable subsets of the interval $T$, and $\lambda$ is Lebesgue measure on $\Re_{\lambda}$ ) to the paths $t \mapsto X_{t}(\omega)$, producing paths $t \mapsto \rho^{\prime}(X .(\omega))(t)$. The main properties of this construction are given in §4; some similar results have been obtained by Itô ([7] and [8]), using classical analysis.

This paper is a revision of a part of the author's 1975 Harvard thesis. The author wishes to thank his advisors, Professors R. M. Dudley and A. M. Gleason, for their comments and encouragement, and to thank Professor A. Bellow (then A. Ionescu Tulcea) for her advice and for pointing out reference [9].

2. Liftings and nonmeasurable processes. We turn to the details of the construction of a complete probability space $(\Omega, Q, P)$, a lifting $\rho$ of $\mathfrak{L}^{\infty}(X, \mathcal{Q}, P)$, and a stochastic process $\left\{X_{t}\right\}_{t \in T}$ on $(\Omega, \mathcal{Q}, P)$ that is Borel measurable but for which the process $\left\{\rho^{\prime}\left(X_{t}\right)\right\}_{t \in T}$ is not Lebesgue measurable.

Let $T=\mathbf{R}$ and let $K=[-\infty, \infty]$. Dudley [2] has shown that if the continuum hypothesis holds, then there is a regular Borel measure $\mu$ on the product space $K^{T}$ such that the process $\left\{\pi_{t}\right\}_{t \in T}$, where $\pi_{t}$ is the projection of $K^{T}$ onto the $t$ th coordinate, has a Borel measurable version, and hence a Borel measurable modification (that every process that has a Borel measurable version has a Borel measurable modification follows, for example, from the main theorem of [3]), but is not itself even Lebesgue measurable. Let $\left\{Z_{t}\right\}_{t \in T}$ be a Borel measurable modification of $\left\{\pi_{t}\right\}_{t \in T}$. Let $\Omega$ be the support of the measure $\mu$, let $\mathcal{Q}$ be the $\sigma$-algebra of subsets of $\Omega$ that belong to the completion under $\mu$ of the Borel $\sigma$-algebra on $K^{T}$, and let $P$ be the restriction to $\mathscr{Q}$ of the completion of $\mu$. Then $(\Omega, Q, P)$ is our probability space. The required process $\left\{X_{t}\right\}_{t \in T}$ is the restriction of $\left\{Z_{t}\right\}_{t \in T}$ to $\Omega$; it is Borel measurable. We shall also need the process $\left\{Y_{t}\right\}_{t \in T}$ formed by restricting the process $\left\{\pi_{t}\right\}_{t \in T}$ to $\Omega$; it is not Lebesgue measurable.

Mokobodzki [9] has shown that if the continuum hypothesis holds, if $S$ is a compact Hausdorff space whose topology has a basis of cardinality at most that of the continuum, if $\nu$ is a regular Borel measure on the $\sigma$-algebra $\mathscr{B}(S)$ of Borel subsets of $S$, and if the support of $\nu$ is $S$, then there is a lifting $\rho$ of $\mathfrak{L}^{\infty}(S, \mathscr{B}(S), \nu)$ that satisfies $\rho(f)=f$ for each continuous real-valued func- 
tion $f$ on $S$. Let $\mathscr{B}(S)^{-}$be the completion of $\Re(S)$ under $\nu$, and let $\nu^{-}$be the completion of $\nu$. It is clear that $\rho$ induces a lifting of $\mathscr{L}^{\infty}\left(S, \mathscr{B}(S)^{-}, \nu^{-}\right)$, again called $\rho$, that satisfies $\rho(f)=f$ for each continuous $f$. It is easy to check that if $K$ is a compact metric space, then the lifting $\rho^{\prime}$ on $\mathscr{R}\left(S, \mathscr{B}(S)^{-}, \nu^{-}, K\right)$ that is induced by $\rho$ satisfies $\rho^{\prime}(F)=F$ for each continuous $K$-valued function $F$ on $S$.

Note that the space $K^{T}$, and hence the space $\Omega$, has a basis whose cardinality is that of the continuum. Hence we can use Mokobodzki's theorem to obtain a lifting $\rho$ of $\mathfrak{L}^{\infty}(\Omega, \mathcal{Q}, P)$ that satisfies $\rho(f)=f$ for each continuous function $f$ on $\Omega$. Let $\rho^{\prime}$ be the lifting of $\Re(\Omega, \mathbb{Q}, P, K)$ induced by $\rho$. Since for each $t$ the random variable $Y_{t}$ is continuous and the random variables $X_{t}$ and $Y_{t}$ satisfy $X_{t} \equiv Y_{t}$, it follows that $\rho^{\prime}\left(X_{t}\right)=\rho^{\prime}\left(Y_{t}\right)=Y_{t}$. Thus the modification of the process $\left\{X_{t}\right\}_{t \in T}$ provided by $\rho^{\prime}$ is the nonmeasurable process $\left\{Y_{t}\right\}_{t \in T}$.

3. The construction of $\rho$-canonical processes. We turn to the application of liftings to the paths $t \mapsto X_{t}(\omega)$, rather than to the random variables $X_{t}$, of the stochastic process $\left\{X_{t}\right\}_{t \in T}$.

Recall that $K$ is a compact metric space. Henceforth we shall suppose that $T$ is a subinterval of $\mathbf{R}$, that $\mathscr{B}(T)$ is the $\sigma$-algebra of Borel subsets of $T$, that $\mathscr{T}_{\lambda}$, or $\mathscr{T}_{\lambda}(T)$, is the $\sigma$-algebra of Lebesgue measurable subsets of $T$, and that $\lambda$ is Lebesgue measure on $T_{\lambda}$.

DEFINITION. Let $\rho$ be a lifting of $\mathcal{L}^{\infty}\left(T, \mathscr{T}_{\lambda}, \lambda\right)$ and let $\rho^{\prime}$ be the associated lifting of $\mathscr{N}\left(T, \Re_{\lambda}, \lambda, K\right)$. Let $\delta_{\rho}$ be the set of all functions $F$ in $\Re\left(T, \Re_{\lambda}, \lambda, K\right)$ for which $\rho^{\prime}(F)=F$. Give $\delta_{\rho}$ the topology of convergence in measure on compact subsets of $T$ (equivalently, the topology defined by the metric

$$
(F, G) \mapsto \int \operatorname{dist}(F(t), G(t)) \frac{1}{1+t^{2}} d t,
$$

where dist is the metric in $K$ ). Finally let $\mathbb{Q}_{\rho}$ be the $\sigma$-algebra on $\delta_{\rho}$ generated by this topology.

Note that $\delta_{\rho}$ contains exactly one function from each equivalence class (under a.e. equality) of measurable functions from $T$ to $K$. Thus the pseudometric defined above is indeed a metric on $\delta_{\rho}$. Also note that a measurable function $F$ from $T$ to $K$ satisfies $\rho^{\prime}(F)=F$ if and only if it satisfies $\rho(\varphi \circ F)=\varphi \circ F$ for each continuous real-valued function $\varphi$ on $K$.

Definition. Let $(\Omega, \mathcal{Q}, P)$ be a probability space and let $\rho$ be a lifting of $\mathfrak{L}^{\infty}\left(T, \mathscr{T}_{\lambda}, \lambda\right)$. A $\rho$-canonical process on $(\Omega, \mathbb{Q}, P)$ is a map from $\Omega$ to $\mathcal{S}_{\rho}$ that is measurable with respect to $\mathbb{Q}$ and $\mathbb{Q}_{\rho}$.

Thus a $\rho$-canonical process is a measurable choice of paths; the paths chosen are "nice" to the extent that functions satisfying $\rho(f)=f$ are nice.

If $X$ is a $\rho$-canonical process, then $X(\omega)$ is a function from $T$ to $K$, and for $t$ 
in $T$ we can consider the map taking $\omega$ to $X(\omega)(t)$; call this map $X_{t}$. Note that the definition of a $\rho$-canonical process does not require that each map $X_{t}$ be measurable; that is, $\left\{X_{t}\right\}_{t \in T}$ is not required to be a stochastic process. However, see Theorem 4.1.

It appears that for some liftings $\rho$ the $\rho$-canonical processes may not be very tractable. The rest of this section is devoted to the construction of liftings for which $\rho$-canonical processes are relatively tractable. The properties of $\rho$-canonical processes are then the subject of the next section.

Definition. Let $t$ be an element of $T$. A sequence $\left\{I_{n}\right\}$ of bounded subintervals of $T$ approaches (or converges to) $t$ if $t$ is an element of each $I_{n}$, and if the length of $I_{n}$ approaches 0 as $n$ approaches $\infty$.

Definition. Let $f$ be a bounded Lebesgue measurable function from $T$ to R. The function $f^{+}$is defined on $T$ by letting $f^{+}(t)$ be the infimum of those real numbers $a$ for which

$$
\lim _{n \rightarrow \infty} \frac{\lambda\left(I_{n} \cap f^{-1}(a, \infty)\right)}{\lambda\left(I_{n}\right)}=0
$$

holds for each sequence $\left\{I_{n}\right\}$ of bounded subintervals of $T$ that converges to $t$. Likewise the function $f^{-}$is defined by letting $f^{-}(t)$ be the supremum of those real numbers $a$ for which

$$
\lim _{n \rightarrow \infty} \frac{\lambda\left(I_{n} \cap f^{-1}(-\infty, a)\right)}{\lambda\left(I_{n}\right)}=0
$$

holds for each sequence $\left\{I_{n}\right\}$ of bounded subintervals of $T$ that converges to $t$.

For each $t$ in $T$, we certainly have $-\infty<f^{-}(t) \leqslant f^{+}(t)<\infty$. The following deeper result will be used later.

LEMMA 3.1. Let $f$ be a bounded Lebesgue measurable function from $T$ to $\mathbf{R}$. Then $f^{-}(t)=f(t)=f^{+}(t)$ for almost every $t$ in $T$.

Proof. For each pair of rational numbers $u, v$ for which $u<v$, let $T(u, v)=\{t \in T: u<f(t)<v\}$. By Lebesgue's differentiation theorem almost every point of $T(u, v)$ is a point of density of $T(u, v)$. If we let $N$ be the union of the countable collection of negligible exceptional sets arising in this way, it follows that $f^{-}(t)=f(t)=f^{+}(t)$ for each $t$ outside $N$.

We need the following calculation of $f^{+}(t)$, which was given by Itô [7]. Since the proof was omitted by Itô, I shall sketch it.

Let $\mathscr{F}_{0}$ be the collection of those nondegenerate compact subintervals of $T$ whose end points are rational or are end points of $T$. Let $\mathscr{F}$ be the collection of finite unions of elements of $\mathscr{F}_{0}$. Certainly every bounded measurable subset of $T$ can be approximated in measure by elements of $\mathscr{F}$.

If $F$ is a bounded nonempty subset of $\mathbf{R}$, define the length of $F$ to be $\sup F-\inf F$. 
LEMMA 3.2. Let $f$ be a bounded Lebesgue measurable function on $T$ and let $t$ be an element of $T$. Then

$$
\begin{aligned}
f^{+}(t)=\sup _{k} \inf _{n} \sup \left\{\frac{1}{\lambda(F)} \int_{F} f(t) d t: F \in \mathscr{F}, t \in F,\right. \\
\text { length } \left.(F) \leqslant \frac{1}{n}, \lambda(F)>\frac{1}{k} \text { length }(F)\right\} .
\end{aligned}
$$

Proof. Certainly $f^{+}(t)$ is the supremum of those real numbers $a$ for which there is a sequence $\left\{I_{n}\right\}$ of bounded subintervals of $T$ that converges to $t$ and satisfies

$$
\varlimsup_{n} \frac{\lambda\left(I_{n} \cap\{f>a\}\right)}{\lambda\left(I_{n}\right)}>0 .
$$

This supremum is equal to the supremum of those real numbers $a$ for which there is a sequence $\left\{E_{n}\right\}$ of bounded nonnegligible measurable subsets of $T$ and a positive number $\varepsilon$ satisfying

$\left(1 / \lambda\left(E_{n}\right)\right) \int_{E_{n}} f(t) d t>a$ for each $n, \lambda\left(E_{n}\right)>\varepsilon \cdot \operatorname{length}\left(E_{n}\right)$

for each $n, t \in E_{n}$ for each $n$, and $\lambda\left(E_{n}\right) \rightarrow 0$ as $n \rightarrow \infty$.

Since arbitrary bounded measurable subsets of $T$ can be approximated by elements of $\mathscr{F}$, this supremum is equal to the number on the right hand side of the equation in the statement of the lemma.

Now we can use $f^{+}$and $f^{-}$to construct liftings $\rho$ for which $\rho$-canonical processes have desirable properties. This construction was suggested by a related construction of $\mathrm{A}$. and C. Ionescu Tulcea (see [6, Proposition 2 of Chapter V]).

THEOREM 3.3. There is a lifting $\rho$ of $\mathfrak{L}^{\infty}\left(T, \mathscr{T}_{\lambda}, \lambda\right)$ such that $f^{-}(t)<\rho(f)(t)$ $\leqslant f^{+}(t)$ holds for each $t$ in $T$ and each $f$ in $\mathfrak{L}^{\infty}\left(T, \mathfrak{T}_{\lambda}, \lambda\right)$.

Proof. For each $t$ in $T$, let $I_{t}=\left\{f^{\sim} \in L^{\infty}\left(T, \Re_{\lambda}, \lambda\right): f^{+}(t)=f^{-}(t)=0\right\}$. Note that if $f$ and $g$ are equal almost everywhere, then $f^{+}(t)=g^{+}(t)$ and $f^{-}(t)=g^{-}(t)$; consequently the definition of $I_{t}$ is meaningful. It is easy to check that each $I_{t}$ is a proper ideal in $L^{\infty}\left(T, \mathfrak{T}_{\lambda}, \lambda\right)$. For each $t$, choose a nonzero algebra homomorphism $\chi$ of $L^{\infty}\left(T, T_{\lambda}, \lambda\right)$ into $\mathbf{R}$ that vanishes on $I_{i}$; that this can be done follows from the elementary theory of complex Banach algebras, once $L^{\infty}\left(T, \mathfrak{T}_{\lambda}, \lambda\right)$ is embedded into the corresponding complex Banach algebra. Now define the function $\rho(f)$ by setting $\rho(f)(t)=$ $\chi_{t}\left(f^{\prime}\right)$. We need to check that $\rho$ is the required lifting. Properties (i)-(iv) of a lifting follow immediately. The following argument shows that $f^{-}(t)<$ $\rho(f)(t) \leqslant f^{+}(t)$ holds for every $f$ and every $t$. Fix $t$ in $T$ and $f$ in $\mathfrak{L}^{\infty}\left(T, \mathscr{T}_{\lambda}, \lambda\right)$. Define a function $g$ on $T$ by setting $g(s)=\max \left(f(s), f^{+}(t)\right)$. On the one hand, $f(s) \leqslant g(s)$ for every $s$, and so $\rho(f)(t)<\rho(g)(t)$. On the other hand, $g^{+}(t)=g^{-}(t)=f^{+}(t)$, from which it follows that $(g-$ 
$\left.f^{+}(t)\right)^{\sim} \in I_{t}$ and hence that $\rho(g)(t)=f^{+}(t)$. Consequently $\rho(f)(t)<f^{+}(t)$. A similar argument shows that $f^{-}(t) \leqslant \rho(f)(t)$. Hence $f^{-}(t) \leqslant \rho(f)(t)<f^{+}(t)$. From this and Lemma 3.1 it follows that $\rho(f) \equiv f$ and hence that $\rho(f)$ is measurable.

Note that if $\rho$ is a lifting of $\mathfrak{L}^{\infty}\left(T, \Re_{\lambda}, \lambda\right)$ that satisfies $f^{-}(t) \leqslant \rho(f)(t) \leqslant$ $f^{+}(t)$ for each $f$ and $t$, then each bounded continuous real-valued function $f$ on $T$ satisfies $\rho(f)=f$; it follows that $\mathcal{S}_{\rho}$ contains all the continuous $K$-valued functions on $T$.

It is of ten convenient to consider paths starting at time $t$ instead of time 0 . This suggests considering along with the sample path $F$ the path $F_{t}$ defined by $F_{t}(s)=F(s+t)$. The following lemma characterizes those liftings for which $\delta_{\rho}$ is closed under such translations. (A lifting $\rho$ of $\mathfrak{L}^{\infty}\left(\mathbf{R}, \mathfrak{T}_{\lambda}, \lambda\right.$ ) or of $\mathfrak{L}^{\infty}\left([0, \infty), \mathfrak{T}_{\lambda}, \lambda\right)$ commutes with translations if $\rho\left(F_{t}\right)=(\rho(F))_{t}$ holds for each $F$ in $\mathfrak{L}^{\infty}\left(\mathbf{R}, \mathfrak{T}_{\lambda}, \lambda\right)$ or in $\mathfrak{L}^{\infty}\left([0, \infty), \mathfrak{T}_{\lambda}, \lambda\right)$ and each $t$ in $\mathbf{R}$ or in $[0, \infty)$.)

LEMMA 3.4. Suppose that the compact metric space $K$ contains at least two points. Let $T$ be $\mathbf{R}$ or $[0, \infty)$, and let $\rho$ be a lifting of $\mathcal{L}^{\infty}\left(T, \Re_{\lambda}, \lambda\right)$. Then $\mathcal{S}_{\rho}$ is closed under each of the translations $F \mapsto F_{t}$ if and only if $\rho$ commutes with translations.

This result is not needed for the main results of this paper; hence its proof, which is straightforward, is omitted.

It is known [4] that there are liftings of $\mathfrak{L}^{\infty}\left(\mathbf{R}, \mathfrak{T}_{\lambda}, \lambda\right)$ that commute with translations, but the following slightly stronger result seems useful in connection with $\rho$-canonical processes.

TheOREM 3.5. Let $T$ be $\mathbf{R}$ or $[0, \infty)$. Then there is a lifting $\rho$ of $\mathfrak{L}^{\infty}\left(T, \mathfrak{T}_{\lambda}, \lambda\right)$ that commutes with translations and satisfies $f^{-}(t) \leqslant \rho(f)(t) \leqslant$ $f^{+}(t)$ for all $t$ in $T$ and all $f$ in $\mathcal{L}^{\infty}\left(T, \Re_{\lambda}, \lambda\right)$.

Proof. First suppose that $T=\mathbf{R}$. Recall the proof of Theorem 3.3 and in particular the characters $\chi_{t}$ of $L^{\infty}\left(T, \Re_{\lambda}, \lambda\right)$. For each $t$ in $T$ define a

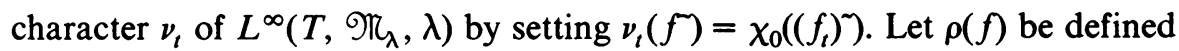
by setting $\rho(f)(t)=\nu_{t}(f)$. Since $f^{-}(0) \leqslant \chi_{0}(f) \leqslant f^{+}(0)$ holds for each $f$ it follows that $f^{-}(t) \leqslant \rho(f)(t) \leqslant f^{+}(t)$ holds for each $t$ and $f$. It follows as in Theorem 3.3 that $\rho$ is a lifting. It is easy to check that $\rho$ commutes with translations.

The proof is similar in case $T=[0, \infty)$; in checking $f^{-}(t)<\rho(f)(t)<$ $f^{+}(t)$ one now uses the relations $f^{-}(t) \leqslant\left(f_{t}\right)^{-}(0)$ and $f^{+}(t) \geqslant\left(f_{t}\right)^{+}(0)$ instead of the relations $f^{-}(t)=\left(f_{t}\right)^{-}(0)$ and $f^{+}(t)=\left(f_{t}\right)^{+}(0)$.

4. The relationship between $\rho$-canonical processes and stochastic processes. We turn to the main results, and consider first the stochastic process induced by a $\rho$-canonical process (Theorem 4.1 ), and then the $\rho$-canonical process induced by a measurable stochastic process (Theorem 4.2). 
THEOREM 4.1. Let $\rho$ be a lifting of $\mathfrak{L}^{\infty}\left(T, \mathfrak{N}_{\lambda}, \lambda\right)$ that satisfies $f^{-}(t)<$ $\rho(f)(t)<f^{+}(t)$ for all $f$ in $\mathfrak{L}^{\infty}\left(T, \Re_{\lambda}, \lambda\right)$ and all $t$ in $T$, let $(\Omega, \mathcal{Q}, P)$ be a complete probability space, and let $X: \Omega \rightarrow \mathcal{S}_{\rho}$ be a $\rho$-canonical process. Let $N=\left\{t \in T: X_{t}\right.$ is not $\mathbb{Q}$-measurable $\}$. Then

(i) the set $N$ is of zero Lebesgue measure,

(ii) the stochastic process $\left\{X_{t}\right\}_{t \in T-N}$ is Lebesgue measurable, and

(iii) if the stochastic process $\left\{X_{t}\right\}_{t \in T-N}$ is continuous in probability, then it is separable. Furthermore, every countable dense subset of $T-N$ is a separating set for $\left\{X_{t}\right\}_{t \in T-N}$.

Proof. First note that if $\varphi$ is a continuous real-valued function on $K$ and if $F \in \mathscr{F}$, then the map $G \mapsto \int_{F} \varphi(G(t)) d t$ is a continuous, hence measurable, function on $\delta_{\rho}$. Consequently for each $F$ in $\mathscr{F}$ the map $\Phi_{F}: \Omega \times T \rightarrow \overline{\mathbf{R}}$ defined by

$$
\Phi_{F}(\omega, t)= \begin{cases}\frac{1}{\lambda(F)} \int_{F} \varphi\left(X_{s}(\omega)\right) d s & \text { if } t \in F, \\ -\infty & \text { if } t \notin F,\end{cases}
$$

is $\mathbb{Q} \times \mathscr{B}(T)$-measurable. Lemma 3.2 implies that

$$
\begin{array}{r}
(\varphi \circ X .(\omega))^{+}(t)=\sup _{k} \inf _{n} \sup \left\{\Phi_{F}(\omega, t): F \in \mathcal{F}, \text { length }(F)<\frac{1}{n},\right. \\
\left.\lambda(F)>\frac{1}{k} \text { length }(F)\right\},
\end{array}
$$

and hence that $(\varphi \circ X .(\omega))^{+}(t)$ is an $\mathcal{Q} \times \mathscr{B}(T)$-measurable function of $(\omega, t)$. From the relation $f^{-}(t)=-(-f)^{+}(t)$, which holds for every $f$ in $\mathfrak{L}^{\infty}\left(T, \Re_{\lambda}, \lambda\right)$ and every $t$ in $T$, it follows that $(\varphi \circ X .(\omega))^{-}(t)$ is also an $\mathbb{Q} \times \mathscr{B}(T)$-measurable function of $(\omega, t)$.

Now Lemma 3.1 implies that for each $\omega$ we have

$$
\left(\varphi \circ X_{.}(\omega)\right)^{-}(t)=\left(\varphi \circ X_{.}(\omega)\right)^{+}(t)
$$

for almost all $t$. Since the functions involved are measurable (with respect to $\mathbb{Q} \times \mathscr{B}(T)$ ), Fubini's theorem implies that the equality holds for almost all $(\omega, t)$.

Since $X .(\omega) \in \mathcal{S}_{\rho}$ we have $\varphi \circ X_{t}(\omega)=\rho(\varphi \circ X .(\omega))(t)$, and so

$$
\left(\varphi \circ X_{.}(\omega)\right)^{-}(t) \leqslant \varphi\left(X_{t}(\omega)\right) \leqslant\left(\varphi \circ X_{.}(\omega)\right)^{+}(t) .
$$

The results of the two preceding paragraphs therefore imply that $(\omega, t) \mapsto \varphi\left(X_{t}(\omega)\right)$ is measurable with respect to $(\mathscr{Q} \times \mathscr{B}(T))^{-}$, the completion of $\mathscr{Q} \times \mathscr{B}(T)$ under $P \times \lambda$. Since the Borel $\sigma$-algebra on $K$ is generated by the functions $\varphi$, it follows that $(\omega, t) \mapsto X_{t}(\omega)$ is itself measurable with respect to $(\mathbb{Q} \times \mathscr{B}(T))^{-}$. The theory of product measures now implies that $\omega \mapsto X_{t}(\omega)$ is $\mathbb{Q}$-measurable for almost every $t$ (recall that $\mathbb{Q}$ is complete under $P$ ). Finally, since $(\omega, t) \mapsto X_{t}(\omega)$ is measurable with respect to $(\mathscr{Q} \times \mathscr{B}(T))^{-}$, its 
restriction to $\Omega \times(T-N)$ is measurable with respect to $\left(\mathscr{Q} \times \mathbb{N}_{\lambda}(T-\right.$ $N))^{-}$. Thus assertions (i) and (ii) are proved.

Now suppose that the process $\left\{X_{t}\right\}_{t \in T-N}$ is continuous in probability. Fix a countable dense subset $D$ of $T-N$. Define the function $H$ on $\Omega \times(T-$ $N)$ by

$$
H(\omega, t)=\lim _{n \rightarrow \infty}\left(\inf \left\{\operatorname{dist}\left(X_{t}(\omega), X_{s}(\omega)\right): s \in D,|s-t|<1 / n\right\}\right),
$$

where dist is the metric in $K$. Since the process $\left\{X_{t}\right\}_{t \in T-N}$ is Lebesgue measurable, $H$ is measurable with respect to $\left(Q \times \mathscr{T}_{\lambda}(T-N)\right)^{-}$. For each $t$ in $T-N$, we can use the fact that $\left\{X_{t}\right\}_{t \in T-N}$ is continuous in probability to choose a sequence $\left\{s_{n}\right\}$ in $D$ approaching $t$ so fast that $\left\{X_{s_{n}}(\omega)\right\}$ approaches $X_{t}(\omega)$ for almost every $\omega$. Thus for each $t$ in $T-N$, we have $H(\omega, t)=0$ almost surely. Fubini's theorem now implies that almost every $\omega$ is in

$$
\Omega_{0}=\{\omega: H(\omega, t)=0 \text { for almost all } t\} .
$$

Now we need only show that for each $\omega$ in $\Omega_{0}$ the graph of the restriction of $X .(\omega)$ to $D$ is dense in the graph of the restriction of $X .(\omega)$ to $T-N$. For this, fix $\omega$ in $\Omega_{0}, t$ in $T-N$, an open interval $I$ containing $t$, and an open neighborhood $U$ of $X_{t}(\omega)$. Choose a continuous real-valued function $\varphi$ on $K$ such that $\varphi\left(X_{t}(\omega)\right)=0$ and $\varphi(x)=1$ if $x \notin U$. Since $X$ is a $\rho$-canonical process, we have

$$
\varphi\left(X_{t}(\omega)\right)=\rho\left(\varphi \circ X_{.}(\omega)\right)(t)
$$

and so $\left(\varphi \circ X_{.}(\omega)\right)^{-}(t) \leqslant \varphi\left(X_{t}(\omega)\right)=0$. Thus for all open intervals $J$ containing $t$ the set $J \cap\left\{s: \varphi\left(X_{s}(\omega)\right)<1\right\}$ has positive Lebesgue measure. Choose such a $J$ that is included in $I$. Since the complement of $\{s: H(\omega, s)=0\}$ is of measure zero, we can choose an element $s$ of $J$ such that $\varphi\left(X_{s}(\omega)\right)<1$ and $H(\omega, s)=0$. Since $H(\omega, s)=0$ and $\varphi\left(X_{s}(\omega)\right)<1$, we can choose $r$ in $J \cap D$ such that $\varphi\left(X_{r}(\omega)\right)<1$. Thus $\left(r, X_{r}(\omega)\right)$ is in the neighborhood $I \times U$ of $\left(t, X_{t}(\omega)\right)$. Since the point $t$ and the neighborhood $I \times U$ are arbitrary, the proof is complete.

THEOREM 4.2. Suppose that $\rho$ is a lifting of $\mathfrak{L}^{\infty}\left(T, \mathfrak{T}_{\lambda}, \lambda\right)$ such that $f^{-}(t) \leqslant$ $\rho(f)(t) \leqslant f^{+}(t)$ holds for all $f$ in $\mathcal{L}^{\infty}\left(T, \Re_{\lambda}, \lambda\right)$ and all $t$ in $T$, and suppose that $(\Omega, \mathcal{Q}, P)$ is a complete probability space. Let $\left\{X_{t}\right\}_{t \in T}$ be a Lebesgue measurable stochastic process on $(\Omega, \mathcal{Q}, P)$ with values in $K$. Then there is a $\rho$-canonical process $Y$ on $(\Omega, Q, P)$ such that $P\left(X_{t}=Y_{t}\right)=1$ holds for almost every $t$. If $Z$ is another $\rho$-canonical process with the same property, then $Y(\omega)=Z(\omega)$ holds for almost every $\omega$ in $\Omega$.

Proof. Since $(\omega, t) \mapsto X_{t}(\omega)$ is $(Q \times \mathscr{B}(T))^{-}$-measurable, the paths $X .(\omega)$ are Lebesgue measurable for almost all $\omega$. For such $\omega$, let $Y(\omega)=\rho^{\prime}(X .(\omega))$. Choose some function $F$ in $\delta_{\rho}$, and let $Y(\omega)=F$ for all other $\omega$. Certainly $Y$ is a function from $\Omega$ to $\delta_{\rho}$. We need to show that it is measurable with respect 
to $\mathbb{Q}$ and $\mathbb{Q}_{\rho}$. Choose a function $U: \Omega \times T \rightarrow K$ that is $\mathbb{Q} \times \mathscr{B}(T)$ measurable and satisfies $U(\omega, t)=X_{t}(\omega)$ for almost all $(\omega, t)$. It is not too hard to check that $\omega \mapsto \rho^{\prime}(U(\omega, \cdot))$ is measurable with respect to $\mathbb{Q}$ and $\mathbb{Q}_{\rho}$ (see, for instance, the proof of Theorem 3 in [1]). However $\rho^{\prime}(U(\omega, \cdot))=$ $Y(\omega)$ for almost all $\omega$, and so $Y$ is measurable. Since both $X_{t}(\omega)$ and $Y_{t}(\omega)$ are $(\mathscr{Q} \times \mathscr{B}(T))^{-}$-measurable functions of $(\omega, t)$, it follows from Fubini's theorem that $P\left(X_{t}=Y_{t}\right)=1$ for almost all $t$.

Finally, if $Y$ and $Z$ are two such $\rho$-canonical processes, then $(\omega, t) \mapsto Y_{t}(\omega)$ and $(\omega, t) \rightarrow Z_{t}(\omega)$ are $(Q \times \mathscr{B}(T))^{-}$-measurable functions such that $P\left(Y_{t}=\right.$ $\left.Z_{t}\right)=1$ for almost every $t$. Then $Y(\omega) \equiv Z(\omega)$ holds for almost every $\omega$, and for each such $\omega$ we have $Y(\omega)=\rho^{\prime}(Y(\omega))=\rho^{\prime}(Z(\omega))=Z(\omega)$.

\section{REFERENCES}

1. D. L. Cohn, Measurable choice of limit points and the existence of separable and measurable processes, Z. Wahrscheinlichkeitstheorie und Verw. Gebiete 22 (1972), 161-165.

2. R. M. Dudley, A counterexample on measurable processes, Proc. Sixth Berkeley Sympos. Math. Statistics and Probability, vol. II, Univ. of California Press, Berkeley, 1972, pp. 57-66; corrections, Ann. Probability 1 (1973), 191-192.

3. J. Hoffman-Jфrgensen, Existence of measurable modifications of stochastic processes, $\mathbf{Z}$. Wahrscheinlichkeitstheorie und Verw. Gebiete 25 (1973), 205-207.

4. A. Ionescu Tulcea and $\mathrm{C}$. Ionescu Tulcea, On the existence of $a$ lifting commuting with the left translations of an arbitrary locally compact group, Proceedings of the Fifth Berkeley Symposium on Mathematical Statistics and Probability, vol. II, Part 1, Univ. of California Press, Berkeley, 1967, pp. 63-97.

5. L Liftings for abstract valued functions and separable stochastic processes, $\mathrm{Z}$. Wahrscheinlichkeitstheorie und Verw. Gebiete 13 (1969), 114-118.

6. Topics in the theory of lifting, Springer-Verlag, New York, 1969.

7. K. Itô, The canonical modification of stochastic processes, J. Math. Soc. Japan 20 (1968), 130-150.

8. , Canonical measurable random functions, Proceedings of the International Conference on Functional Analysis and Related Topics, Tokyo, April, 1969, Univ. of Tokyo Press, Tokyo, 1970, pp. 369-377.

9. G. Mokobodzki, Relèvement borélien compatible avec une classe d'ensembles négligeables. Application à la désintégration des mesures, Lecture Notes in Math., vol. 465, Springer-Verlag, Berlin and New York, 1975, pp. 437-442.

Department of Mathematics, Suffolk University, Boston, Massachusetts 02114 